\title{
Secondary Intra-Abdominal Pregnancy: A Case Report
}

\author{
Shanbhag A, Singh A \\ Department Obs/Gyn, Manipal Teaching Hospital, Pokhara, Nepal
}

\begin{abstract}
Abdominal pregnancy is extremely rare, and has been historically defined as an implantation in the peritoneal cavity, exclusive of tubal, ovarian and intraligamentary pregnancy. Abdominal pregnancy can be primary or secondary. The presence of abdominal pregnancy is associated with increased maternal and perinatal morbidity and mortality. The reported incidence of abdominal pregnancy ranges from 1 per 2200 to 1 per 10,200 pregnancies 1 . The early recognition and management of the same is necessary to prevent untoward complications. This case report describes an interesting case of a 16 year primigravida with a live secondary abdominal pregnancy.
\end{abstract}

Keywords: Abdominal pregnancy, hemoperitoneum, methotrexate

\section{INTRODUCTION}

Abdominal pregnancy is extremely rare, and has been historically defined as an implantation in the peritoneal cavity, exclusive of tubal, ovarian and intraligamentary pregnancy. The presence of abdominal pregnancy is associated with increased maternal and perinatal morbidity and mortality. It can be primary or secondary, with the latter being more common. Approximately $1 \%$ of all pregnancies are extra uterine, and $1 \%$ to $3 \%$ of these are peritoneal implantations1. The reported incidence of abdominal pregnancy ranges from 1 per 2200 to 1 per 10,200 pregnancies1. Advanced abdominal pregnancy is rare with a reported incidence of 1 in 22,000 pregnancies 1 . The risk factor for abdominal pregnancy is the same as ectopic pregnancy, and when it is recognized, immediate removal of the fetus from the peritoneal cavity is recommended. We report a case of live secondary abdominal pregnancy of 18 weeks of gestation.

\section{CASE}

An un-booked case of 16 year old primigravida, presented for the first time at 18 weeks period of gestation, with complaints of abdominal pain for one week. There was no history of bleeding per vagina, dizziness or fever.

On examination, patient was pale. Pulse rate was 96 per minute, blood pressure was $90 / 60 \mathrm{mmHg}$.

\section{CORRESPONDENCE}

Dr. Asha Shanbhag, MS, D Mas, F Mas

Department of Obstetrics and Gynaecology

Manipal Teaching Hospital

PO Box 341, Phulbari Pokhara, Nepal

Telephone: 0097761 526416; 09845391704

Email: ashoo_sdr@yahoo.co.in
Per abdomen, examination revealed a distended abdomen with diffused tenderness. Hemoglobin : $5.0 \mathrm{gm} / \mathrm{dl}$, platelet count and coagulation profile were within normal limits. On USG Uterus was bulky, of normal echo texture with empty endometrial cavity. A single live fetus corresponding to 18 weeks period of gestation, with placenta was seen in the peritoneal cavity. There was no myometrium seen around the fetus or the placenta. Large amount of blood collection was seen in the peritoneal cavity.

On Emergency laparotomy opening abdomen by sub-umbilical vertical incision, about one litre of hemoperitoneum was present. Uterus was of normal size. The site of an old ruptured ectopic pregnancy was noted on the right tube. Placenta was seen attached to the ruptured site of right tube and partly over the omentum. Fetus was covered by the amniotic membrane but spilled amniotic fluid was seen in the abdominal cavity.

Right-sided salpingectomy was done along with partial omentectomy. Hemostasis was achieved. Drainage tube was placed, which was removed on the second postoperative day. The patient received three pints of blood, intra- operatively. Her postoperative period was uneventful. 


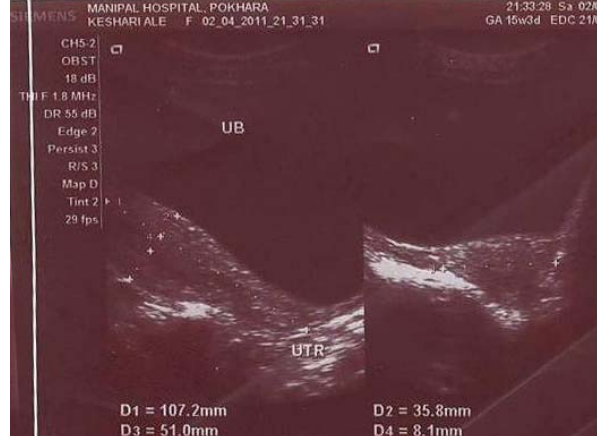

Figure 1. Ultrasound showing an empty uterine cavity

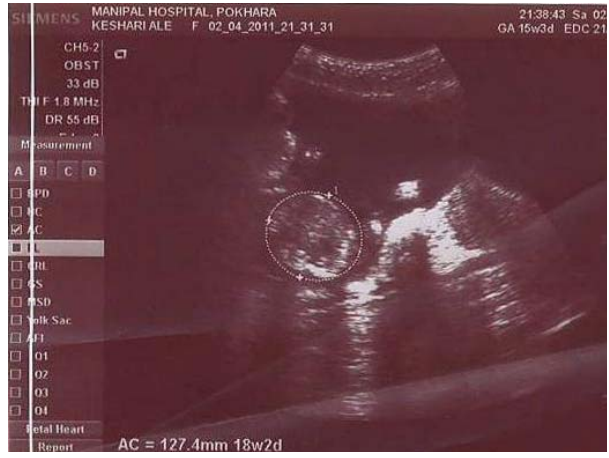

Figure 2. Fetus of $18+$ weeks lying freely in the abdominal cavity

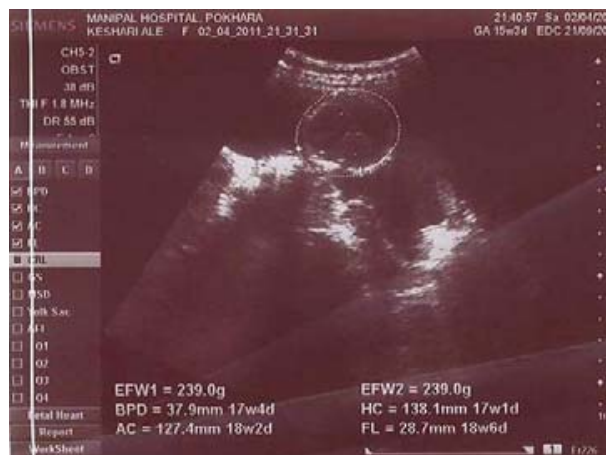

Figure 3. Anthropometric measurement showing fetus of 18 weeks

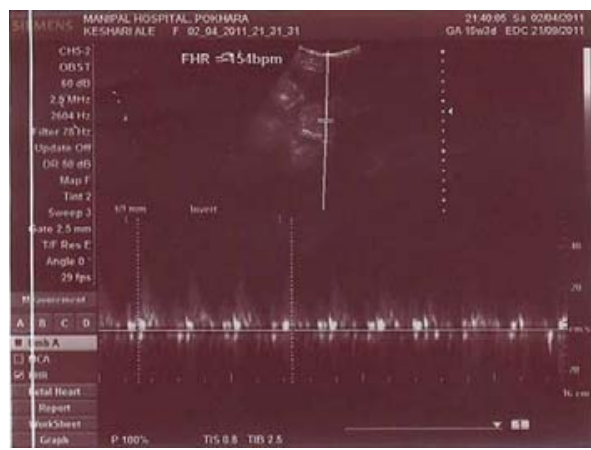

Figure 4. Doppler showing cardiac activity of the intra abdominal fetus

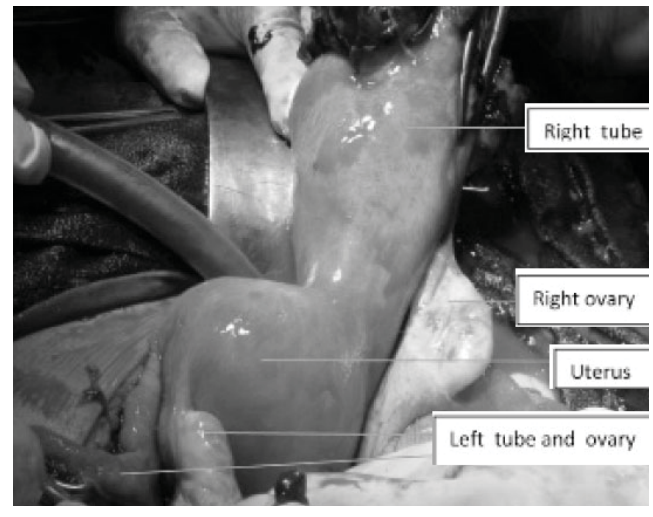

Figure 5. Uterus with an intact left tube and ovary, with the ruptured right fallopian tube

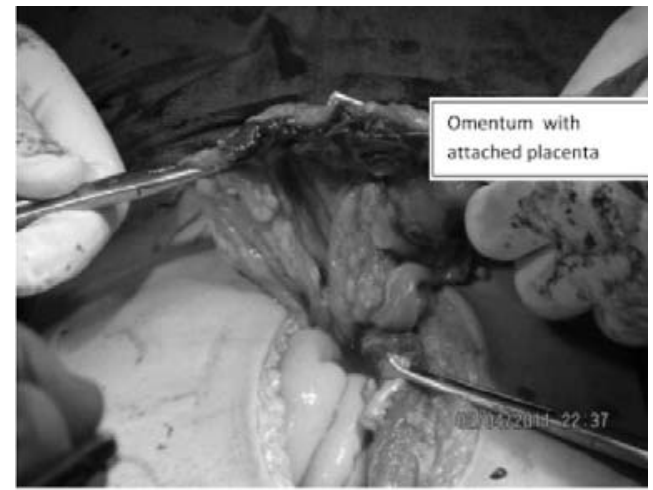

Figure 6. Omentum with the placenta adherent onto it

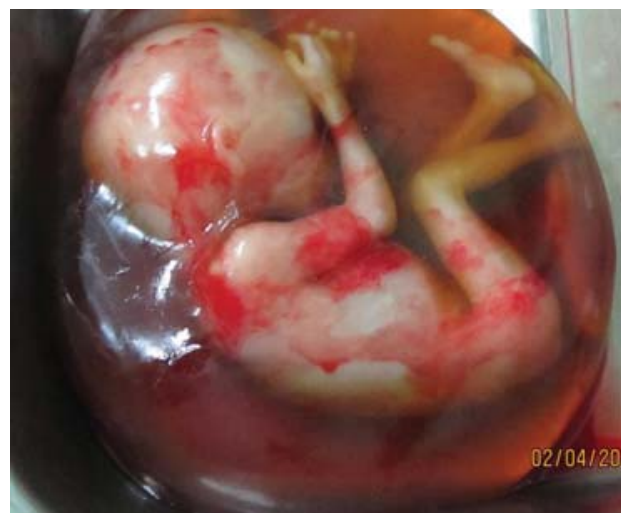

Figure 7. Fetus within an intact amniotic sac 


\section{COMMENT}

Approximately $50 \%$ of ectopic pregnancies are missed at the time of initial presentation. Abdominal pregnancy is potentially life-threatening, with maternal and perinatal mortality rates of $0.5 \%$ to $18 \%$ and $40 \%$ to $95 \%$, respectively ${ }^{2}$. Rarely, the embryo is expelled into the abdomen and the afterbirth remains attached to the tube and the embryo continues to live and grow. Such a condition is referred to as a secondary abdominal pregnancy. Primary abdominal pregnancy in which the fertilized egg attaches to an abdominal organ and ovarian pregnancies are rarer still. Ultrasound, MRI, CT scan and laparotomy are tools to distinguish between primary and secondary abdominal pregnancies. The patient with abdominal pregnancy typically presents with constant abdominal pain, progressive anemia and sudden loss of fetal movements.

During laparotomy the clinician is the one to make a decision regarding the fate of the placenta. Postoperative maternal morbidity will be lessened with the complete removal of the placenta, provided it is technically feasible. If vascular attachment involves major blood vessels or vital structures, the organ should be left undisturbed. 3 Retention of the placenta in situ is not without risks and postoperative morbidity. Postoperative methotrexate has been administered by some for resorption of the placenta, but it leads to accumulation of necrotic debris due to accelerated placental resorption and associated morbidity.4 Secondary hemorrhage, abscess formation, paralytic ileus and bowel obstruction, have all been reported as the complications of leaving the placenta in situ. Resorption of placenta is a slow process and can be monitored by serial ultrasound, CT scan or MRI.

However, there is no definite consensus regarding the management of the placenta, and each case should be individualized based on the intra operative findings. In our patient, the majority of placenta was attached to the fallopian tube, and only a part of it was attached to the omentum, and thus the complete removal of the placenta was feasible (Figure 1 to 7 ).

\section{CONCLUSIONS}

Proper preoperative evaluation, use of systemic Methotrexate, assurance of sufficient blood products, availability of a multidisciplinary surgical team and proper operative techniques in managing abdominal pregnancy can reduce maternal morbidity and mortality.

\section{REFERENCES}

1. Parekh VK, Bhatt S, Dogra VS. Abdominal pregnancy: An unusual presentation. J Ultrasound Med.2008;27:679-81.

2. Abbott J, Emmans LS, Lowenstein SR. Ectopic pregnancy: Ten common pitfalls in diagnosis. Am J Emerg Med.1990;8:515-22.

3. Hallatt JG, Grove JA. Abdominal pregnancy: A study of twenty-one consecutive cases. Am J Obstet Gynecol.1985 Jun 15;152(4):444-9.

4. Cunningham GF, Levine KJ, Bloom SI. William obstetrics. 22nd ed. London: Prentice Hall International (UK); 2005. p.255-56.

5. Ramachandran K, Kirk P. Massive haemorrhage in a previously undiagnosed abdominal pregnancy presenting for elective cesarean delivery. Can J Anaesth.2004;51:57-61. 